\title{
104 \\ GraphApp: A High-Level Toolkit for Building Prototype User Interfaces
}

\author{
Lachlan J. Patrick \\ Basser Department of Computer Science \\ University of Sydney \\ AUSTRALIA \\ loki@cs.su.oz.au
}

\begin{abstract}
GraphApp is a cross-platform high-level toolkit for the construction of graphical interfaces to applications. It has been designed specifically to be easy and quick to learn, and was used in a course teaching user interface design. This paper gives a brief outline of the motivations for the creation of this toolkit and the approaches taken to achieve the design goals. A brief summary of student usage of the toolkit is presented.
\end{abstract}

KEYWORDS prototyping, building user interfaces, teaching user interface design, cross-platform user interface toolkit

\section{INTRODUCTION}

There have been few graphics toolkits which have been designed specifically with learning in mind. The SUIT toolkit (Pausch, 1992) is one; Tk (Ousterhout, 91 ) is another which is sometimes used for teaching, despite not being made especially for beginners.

A graphics toolkit designed for learners should satisfy a number of conditions. It must be easy to learn and be sufficiently powerful to use in real-world programs. Good documentation is also important for learners.

GraphApp (Patrick, 1996) was used in a course teaching user interface design to computer science students, which introduces a number of stricter requirements for the design to satisfy.

The toolkit should be representative of event-driven graphics toolkits in general, so that students can transfer the lessons they learn to other systems. If it works on multiple operating systems, this is advantageous since students can take source code home and experiment.
Additionally, making programs easy to understand and fast to learn are important factors in a course with only a brief time allotted for teaching interface construction tools.

GraphApp met all of these design goals and was used with much success in the above-mentioned course, which also introduced students to HTML, Tk and Visual Basic.

This paper describes some of the design decisions made during GraphApp's development, and briefly describes its use in teaching.

\section{THE DESIGN OF GRAPHAPP}

GraphApp is a real-world, cross-platform, easy to learn graphics toolkit with a vocabulary and design optimised for user interface construction.

\subsection{Cross-platform}

GraphApp works on multiple platforms to facilitate the transfer of source code between the workplace and the home computer, or between different workplace

Human-Computer Interaction: INTERACT'97 S. Howard, J. Hammond \& G. Lindgaard (editors) Published by Chapman \& Hall CIFIP 1997 
computers. It implements many of the most common widgets available in modern graphical operating systems, is small and efficient and the source code is readily available.

The $C$ language (Kernighan, 1988) was chosen as the target language for the toolkit, since this is a common, standard language for which compilers exist on most operating systems. Porting GraphApp was thus not impeded by language choice.

The students who were taught GraphApp as part of a third year computer science course already had a familiarity with the $C$ language, and prior surveys had shown that many owned PC compatible computers at home. The practical part of the course made use of XWindows terminals linked to a central file server.

GraphApp was useful in this course because it allows source code portability between $\mathrm{X}$-Windows and Microsoft Windows (Petzold, 1992) platforms. The library is layered above each operating system so as to give access to widgets using the native appearance of each platform.

\subsection{Easy to Learn}

GraphApp is easy to learn for a number of reasons: the use of sensible defaults; initialisation and event handling on demand; short parameter lists; and sensible identifiers are all factors.

The use of sensible defaults is an example of how 'training wheels' (Carroll, 1984) can improve learning. The student need not know how to use colours, fonts or windows to begin programming, but these concepts can be learned as experience increases.

There is no need to explicitly initialise the library or write event handling routines, because this is all performed automatically as needed.

Function parameter lists have been kept short through the strategic use of a small number of global variables, the passing of structures in some cases, and appropriate function naming.

The use of verbs as function names reduces some parameter lists, simplifying learning and providing a better match between student expectations and GraphApp code than would be the case with some other systems. For instance there are polymorphic functions named 'show' and 'hide', which affect the visibility of graphical objects, rather than a single 'showhide' function which takes an extra parameter.

\section{IN THE CLASSROOM}

GraphApp was employed as part of a course teaching user interface design, and the response to the toolkit was positive. It was used both in a small exercise and a major task by many students.

Students were introduced to HTML, Tk, Visual Basic and GraphApp, and were given two one-hour lectures and four hours of practical work to learn each tool. The aim was for all students to be able to complete a small exercise by the end of that time, so each tool had to be easy to learn.

For a major assessment task, students chose one of the tools and extended the exercise task into a full program. GraphApp was used by almost $40 \%$ of students for this project, with HTML being more highly favoured at almost $50 \%$.

The fact that GraphApp was often used is an encouraging sign. A survey of the perceived relevance of these tools was also conducted, and the $70 \%$ of the class who responded ranked the above tools in the same order as their use in the major assessment task.

\section{CONCLUSION}

GraphApp is a cross-platform graphics toolkit with a vocabulary and design optimised for learning, which has been used successfully in a course teaching user interface design.

\section{REFERENCES}

Carroll, J.M.; Carrithers, C.; "Training Wheels in a User Interface", Comm. of the ACM, Vol. 27, No. 8, (August 1984) pp 800-806

Kernighan, B.; Richie, D.; The C Programming Language, 2nd ed., Prentice Hall, (1988)

Ousterhout, J. K.; "An X11 Toolkit Based on the

Tcl Language,” USENIX, (Winter 1991)

Pausch, R.; Conway, M.; DeLine, R.; "Lessons Learned from SUIT, the Simple User Interface Toolkit", ACM Trans. on Information Systems, Vol. 10, No. 4, (October 1992), pp 320-344

Patrick, L.; http://www.cs.su.oz.au/ loki/graphapp

Petzold, C.; Programming Windows 3.1, 3rd ed., Microsoft Press, (1992) 